\title{
Enfermedad cardiovascular: creencias y prácticas en la adherencia al tratamiento
}

\author{
Cardiovascular disease: beliefs and practices in the adherence to the treatment \\ Doença cardiovascular: crenças e práticas na aderência ao tratamento
}

Olga Patricia Melo-Barbosa*

\begin{abstract}
Autor de correspondencia
* $\triangle$ Enfermera Epidemióloga, M.Sc en Educación, Candidata a Ph.D en Ciencias Sociales y Humanas PUJ. Docente investigadora Universidad Manuela Beltrán y Universidad Antonio Nariño. e-mail: patrymelobar@ uan.edu.co. (D) https://orcid.org/0000-00022465-3837.
\end{abstract}

Este es un artículo bajo la licencia CC BY

(https://creativecommons.org/ licenses/by/4.0/) @ (1)

\section{Resumen}

Introducción: Las enfermedades cardiovasculares representan a nivel global un problema de salud pública de dimensiones importantes después de las enfermedades infecciosas; de ahí que para que la morbimortalidad de estas enfermedades disminuya se hace necesario que los pacientes se adhieran a los tratamientos. Dentro de los factores de no adherencia se encuentran las creencias respecto a la enfermedad o su tratamiento, que generalmente están influenciadas por el entorno social y cultural. Metodología: Se realizó una revisión de la literatura en las bases de datos EBSCO HOST, PUB-MET, DIALNET, Wolters Kluwer Ovid, LILACS, SCIELO, ScienceDirect Medline y Pubmed. Los artículos se eligieron teniendo en cuenta que fuesen publicados entre enero de 1990 a diciembre de 2016, con los siguientes términos de búsqueda: creencias en tensión arterial, belief cardiovasculares diseases, creencias y prácticas en salud cardiovascular, creencias sobre la enfermedad cardiovascular, adherencia al tratamiento de la enfermedad cardiovascular, beliefs in cardiovascular diseases, adherence treatment disease cardiovascular. De los artículos seleccionados: 330 se escogieron por el título, 129 se excluyeron porque el título no es pertinente para el objeto de esta revisión y 51 fueron recuperados para lectura crítica. Resultados: Se encontró que los pacientes tienen muchas y variadas creencias frente a la enfermedad y su tratamiento; razón por la cual utilizan prácticas alternativas para el manejo de la enfermedad, que van desde la acupuntura, infusiones de plantas, recetas caseras hasta el chamanismo. Conclusiones: El enfermero está llamado a identificar a los pacientes no adherentes, a investigar acerca de las creencias y prácticas para diseñar intervenciones más efectivas y eficientes teniendo en cuenta el cuidado transcultural.

Palabras clave: Cuidado transcultural, creencias, enfermedades cardiovasculares, adherencia.

\footnotetext{
${ }^{\dagger}$ El título sugiere el abordaje de la enfermedad cardiovascular en general, al hacer la revisión de la literatura con las palabras claves relacionadas se evidenció que las personas presentan más creencias y prácticas con la hipertensión arterial.
} 


\begin{abstract}
Introduction: Cardiovascular diseases represent, globally, a public health problem of important dimensions after infectious diseases; for the morbimortality of these diseases to decrease, its necessary that the patients adhere to the treatments. Within the factors of non-adherence are the beliefs regarding the disease or its treatment, which generally are influenced by the social and cultural environment. Methodology: A review of the literature was performed in the data bases EBCO HOST, PUB-MET, DIAL-NET, Wolters Kluwer Ovid, LILACS, SCIELO, ScienceDirect Medline and Pubmed. The articles were selected considering the date of publication from January 1990 to December 2016, with the following search terms: beliefs in blood pressure, cardiovascular disease beliefs, beliefs and practices of cardiovascular health, beliefs about cardiovascular disease, adherence to treatment of cardiovascular disease, beliefs in cardiovascular disease. From the articles selected, 330 were chosen by its title, 129 were excluded because the title is not relevant for the objective of this review, and 51 were recovered for critical review. Results: It was found that the patients have many and diverse beliefs regarding the disease and its treatment; reason why patients use alternative practices for the treatment of the disease, these go from acupuncture, herbal infusions, home recipes to shamanism. Conclusions: The nurse is required to identify non-adherent patients, and to research about the beliefs and practices to design more effective and efficient interventions considering transcultural care.
\end{abstract}

Keywords: transcultural care, beliefs, cardiovascular diseases, adherence.

\begin{abstract}
Resumo
Introdução: As doenças cardiovasculares representam globalmente um problema de saúde pública de dimensões importantes depois das doenças infecciosas; sendo que para que a morbimortalidade destas doenças diminua faz-se necessário que os pacientes se tenham aderido aos tratamentos. Dentro dos fatores de não aderência se encontram as crenças respeito à doença ou seu tratamento, que geralmente estão influenciadas pelo ambiente social e cultural. Metodologia: Realizou-se uma revisão da literatura nas bases de dados EBSCO HOST, PUB-MET, DIALNET, Wolters Kluwer Ovid, LILACS, SCIELO, ScienceDirect Medline e Pubmed. Os artigos foram escolhidos levandose em conta que foram publicados entre janeiro de 1990 a dezembro de 2016, com os seguintes termos de pesquisa: crenças em pressão arterial, belief cardiovasculares diseases, crenças e práticas em saúde cardiovascular, crenças sobre a doença cardiovascular, aderência ao tratamento da doença cardiovascular, beliefs in cardiovascular diseases, adherence treatment disease cardiovascular. Dos artigos selecionados: 330 se escolheram pelo título, 129 se excluíram porque o título não era pertinente para o objeto desta revisão e 51 foram recuperados para leitura crítica. Resultados: Encontrou-se que os pacientes têm muitas e variadas crenças frente à doença e seu tratamento; razão pela qual utilizam práticas alternativas para o manejo da doença, que vão desde a acupuntura, infusões de plantas, receitas caseiras até o xamanismo. Conclusões: $\mathrm{O}$ enfermeiro está chamado a identificar os pacientes não aderentes, a pesquisar sobre as crenças e práticas para criar intervenções mais efetivas e eficientes tendo em conta o cuidado transcultural.
\end{abstract}

Palavras-chave: Cuidado transcultural, crenças, doenças cardiovasculares, aderência.

\section{Introducción}

En el Informe del 2015 sobre la salud en el mundo publicado por la Organización Mundial de la Salud -OMS- las Enfermedades Cardiovasculares -ECVocupan el primer lugar con 10.000 mil defunciones; le siguen las Neoplasias Malignas con 4000, de las cuales una gran cantidad son de países con ingresos bajos. En Colombia de 30.000 muertes en 1980 pasó a 55.000 en el 2004 (1). En tal sentido, este tipo de enfermedades se ubica en los primeros lugares de mortalidad y morbilidad, siendo una tendencia creciente dado su carácter crónico; además, es un grave problema de salud pública, que aumenta su prevalencia, así como sus complicaciones y costos. Por su parte, la Organización Panamericana de la Salud -OPS- conjuntamente con el Instituto Nacional de Salud en su informe anual de indicadores básicos de la Situación de Salud en 
ISSN-PRINT

1794-9831

E-ISSN 2322-7028

Vol. 15 No. 2

Jul - Dic 2018

Cúcuta, Colombia
Colombia para el año 2010 (2) y con los resultados del DANE, presentaron los indicadores causantes de mortalidad por enfermedades crónicas con un total de 27.822 muertes y una tasa de 263,7 tomando como base la tasa de 100.000 habitantes; es decir, que existe un alto número de muertes por enfermedad Isquémica del Corazón en personas de 45 y más años.

Las evidencias anteriores muestran el grave problema de salud pública que representan las enfermedades cardiovasculares; por esta razón y según el análisis realizado por la OMS es necesario que los pacientes que tienen algún tipo de tratamiento farmacológico y no farmacológico, lo sigan a conciencia, sin modificar las prescripciones; asimismo, deben obedecer los consejos sobre estilos de vida saludables, como la dieta, el ejercicio, y la medicación. En los países desarrollados la adherencia terapéutica en pacientes que padecen enfermedades crónicas es del $50 \%$ y se supone que esta deficiencia sea aún mayor en países en desarrollo. Así mismo, con la hipertensión arterial, el porcentaje de pacientes que cumple su régimen terapéutico es sólo el $27 \%$ en Gambia, $43 \%$ en China y $51 \%$ en Estados Unidos (3).

La no adherencia a los tratamientos trae muchas y variadas consecuencias entre las que se encuentran consecuencias médicas o clínicas tales como: retrasos en la curación, recaídas, aparición de complicaciones y riesgo de aumentar el desarrollo de resistencias medicamentosas. Con relación a las consecuencias económicas se presentan altos gastos farmacéuticos, ausentismo laboral por parte del paciente, alojamiento innecesario de medicamentos no consumidos en el hogar, con el riesgo de que los niños se intoxiquen; a nivel sanitario se incrementa la atención médica desaprovechada, aumento de intervenciones y hospitalizaciones e incremento del uso de los servicios (4). Por todo lo anterior, es importante saber por qué los pacientes con ECV no se adhieren a los tratamientos. En consecuencia, el objetivo de este trabajo es presentar una revisión de la literatura relacionada con las creencias y prácticas respecto a la adherencia al tratamiento farmacológico y no farmacológico en adultos con enfermedad cardiovascular.

Ahora bien, la OMS (5) proporciona un análisis crítico sobre la no adherencia a los tratamientos en pacientes con enfermedades crónicas; sin embargo, recomienda reforzar la investigación sobre la adherencia terapéutica; de modo similar, reconoce los beneficios al abordar la adherencia para los pacientes y la sociedad en general. Desde esta perspectiva, es necesario e importante investigar sobre las creencias y prácticas de estos pacientes frente a su enfermedad, ya que es necesario que el enfermero (a) investigue estos comportamientos para planificar acciones más efectivas y eficientes.

\section{Metodología}

Se hizo una revisión de la literatura, para lo cual se consultaron las siguientes bases de datos: EBSCO HOST, PUB-MET, DIALNET, Wolters Kluwer Ovid, LILACS, SCIELO, ScienceDirect Medline y Pubmed. Los artículos se eligieron teniendo en cuenta que fuesen publicados entre enero de 1990 a diciembre de 2016 , con los siguientes términos de búsqueda: creencias en tensión arterial, belief cardiovasculares diseases, creencias y prácticas en salud cardiovascular, creencias en enfermedad cardiovascular, comportamientos en hipertensión arterial, adherencia al tratamiento enfermedad cardiovascular, beliefs in cardiovascular diseases, Adherence treatment disease cardiovascular. De acuerdo a los criterios mencionados anteriormente se tuvieron en cuenta: 330 artículos que fueron seleccionados por título, 129 se excluyeron porque el título no es pertinente para el objeto de esta revisión, 110 se excluyeron después de la lectura del resumen y 40 con la lectura del cuerpo del artículo, finalmente 51 artículos fueron recuperados para lectura crítica.

\section{Resultados}

Esta investigación fue realizada teniendo en cuenta estudios elaborados en países como Estados Unidos, Austria, Reino Unido, Irlanda; es de hacer notar que se encuentra bastante literatura en Brasil con 13 estudios, seguido de España con 10, Colombia con 5 y Cuba con 4. Dentro de la literatura consultada el enfoque que más predominó fue el cuantitativo con 32 estudios, le sigue el cualitativo con 14, 4 revisiones sistemáticas y 1 estudio cuali-cuantitativo. Para la contextualización de esta revisión es necesario definir los conceptos que atañen a este estudio, el primero es el de Adherencia. En el informe presentado por la OMS (6) en el 2004, sobre adherencia a los tratamientos a largo plazo, se la define como el grado en que el paciente sigue las instrucciones médicas; en este mismo informe se reflexiona acerca del papel que tiene el paciente en su tratamiento, por el cual las instrucciones conllevan a que se considere al paciente, no como un agente activo en el proceso de tratamiento, sino como un actor pasivo. La adherencia 
no sólo habla de si los pacientes se toman o no los medicamentos, esta definición abarca muchas más actividades, relacionadas con la búsqueda de atención médica, conseguir el medicamento recetado, tomar la medicación apropiadamente, cumplir con las consultas de seguimiento y ejecutar las modificaciones en los comportamientos que tienen que ver con el régimen alimentario, el tabaquismo, el alcohol y la actividad física.

También es importante tener en cuenta, que la no adherencia a los tratamientos ocasiona trastornos psicosociales y económicos, así como consecuencias médicas o clínicas tales como las recaídas (7). Los factores que interfieren en la adherencia van desde la difícil comunicación efectiva entre el profesional y el paciente, la alta frecuencia del uso de términos técnicos por los profesionales de la salud cuando hablan con los pacientes, que el personal de salud pase menos de dos minutos dando información del tratamiento al paciente, facción por la interacción personal de saludpaciente. Sin embargo, se debe precisar que el personal de salud sobreestima la importancia del tiempo que emplea dando información al paciente y subestima el deseo de los pacientes para obtener información (8).

El segundo término es el de creencias. Según la Real Academia Española, la palabra creer viene del latín creděre, que significa tener por cierto algo que el entendimiento no alcanza o que no está comprobado o demostrado. En su primera acepción define la creencia como firme asentimiento y conformidad con algo. Y el tercero, son las prácticas. La Real Academia de la Lengua define la palabra práctica así:

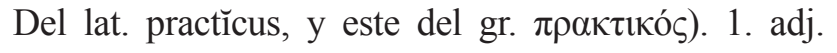
Perteneciente o relativo a la práctica. 2. adj. Se dice de los conocimientos que enseñan el modo de hacer algo. 3. adj. Experimentado, versado y diestro en algo. 4. adj. Que piensa o actúa ajustándose a la realidad y persiguiendo normalmente un fin útil (9). Para fines de esta revisión la palabra práctica se refiere a las prácticas, acciones, comportamientos que los pacientes realizan para el manejo de su enfermedad.

Se relacionan las ocho categorías emergentes después de la lectura crítica de los 51 artículos. Posteriormente se justifica cada una de ellas con los artículos encontrados.

\section{Adherencia al tratamiento en ECV}

Se ha documentado ampliamente que la adherencia a los tratamientos farmacológicos y no farmacológicos de los pacientes con ECV o crónica es muy pobre; en promedio la adherencia para estas patologías es del $50 \%, 44.9$ (10), cerca del $40 \%$ para el tratamiento farmacológico y entre el $60-90 \%$ en las medidas higiénico-dietéticas. Granados et al. (11) hacen referencia a las estadísticas de la adherencia en la Hipertensión Arterial, señalando que el porcentaje de pacientes hipertensos que no siguen adecuadamente las instrucciones del personal sanitario oscila entre el $20 \%$ y el $80 \%$, con un promedio de $50 \%$.

La adherencia se ve afectada por las creencias que los pacientes tienen respecto a su enfermedad (1217). Una gran cantidad de pacientes se adhieren a los tratamientos según los síntomas que creen tener de su enfermedad, un ejemplo claro es en el caso de pacientes con hipertensión arterial; en este caso, algunos pacientes se toman el medicamento si tienen cefalea, mareos o sudoración, si estos síntomas no se presentan no es necesario tomarse el medicamento (18). También a las prácticas que los pacientes realizan como tratamientos alternativos para tratar su enfermedad; algunos pacientes usan recetas caseras para el manejo de su enfermedad, por ejemplo toman té de alpiste, la oliva, el té, las pastillas de ajo, o van a terapias alternativas como herbolaria, masoterapia, homeopatía, magnetoterapia y acupuntura (19).

\section{Creencias en ECV}

Es sabido que enfermedades como la Hipertensión Arterial no presentan signos ni síntomas en su semiología, algunos la han denominado el enemigo silencioso. Brondolo et al., 1999; Fasce, Flores y Fasce, 2002; Kaplan y Lieberman, 1999-, en sus estudios hablan de los signos y síntomas que los pacientes refieren respecto a su enfermedad, sobre todo con la Hipertensión Arterial (20-22) y la Insuficiencia Cardiaca. Estos estudios evidencian que los pacientes hipertensos que dicen percibir síntomas (pacientes con creencias en síntomas asociados a la enfermedad), se comportan ante la enfermedad hipertensiva como si se tratara de una enfermedad sintomática, cíclica o recurrente.

Por otra parte, Roales et al. (23) demuestran que el $81,3 \%$ de los pacientes manifestaron reconocer cuándo su Tensión Arterial está elevada en base a los síntomas que perciben; estos síntomas son: cefaleas, mareos, nerviosismo-inquietud, rubefacción-sofocos, 
ISSN-PRINT

1794-9831

E-ISSN 2322-7028

Vol. 15 No. 2

Jul - Dic 2018

Cúcuta, Colombia palpitaciones-taquicardia, debilidad, vista nublada, presión en el pecho, náuseas-vómitos, agitaciones (24). Algunos pacientes perciben la Tensión Arterial como de aparición súbita y acompañada de síntomas y complicaciones que les causa gran dependencia y requiere de atención médica urgente; entre los síntomas que dicen sentirse encuentran: edema de extremidades y cefaleas. Así mismo, sólo ingieren medicamentos cuando tienen síntomas que los incomodan.

En el estudio de Mendoza et al. (25) los pacientes del estudio consideraron que las ECV causan muerte súbita, que son producto de acontecimientos dramáticos, como crisis nerviosas y enojo, estrés; del mismo modo, otros estudios hablan de estrés por racismo, violencia en las calles o incluso por estar muy feliz. Un tema común entre los grupos fue el temor a la violencia y la delincuencia de las comunidades; es decir, que estos problemas sociales serían responsables de gran parte de las enfermedades cardiovasculares. También creen o manifiestan que las enfermedades cardiovasculares sólo afecta a personas mayores de 30 años, y más a las mujeres que a los hombres por todos sus roles en la sociedad; a su vez los hombres dijeron que eran más afectados por las enfermedades cardiovasculares que las mujeres debido al esfuerzo que deben realizar en trabajos pesados y la tensión resultante de la responsabilidad de mantener a sus familias.

Otro aspecto importante en las creencias que tienen los pacientes de la enfermedad cardiovascular, es que estas enfermedades tienen menor relevancia que otras patologías en las mujeres. Jensen y sus colegas reportaron que sólo el $13 \%$ de las mujeres de la muestra fueron capaces de identificar las enfermedades del corazón como la principal causa de muerte en las mujeres a pesar de las múltiples campañas a la comunidad; la mayoría de las mujeres $(51 \%$ a 55 $\%$ ) estaban preocupadas por el cáncer o el cáncer de mama como mayor riesgo para la salud; Frick y sus colegas informaron que sólo el $40 \%$ de las mujeres se consideraban bien o muy bien informado sobre las enfermedades del corazón (26).

\section{Creencias frente al tratamiento en ECV}

Algunos pacientes han referido sobre su experiencia al tener que tomar la pastilla el resto de su vida: $-\mathrm{Me}$ mantengo gracias a la pastilla por la mañana... y eso es para toda la vida-, -...fue mandarme las pastillas y se me quitó todo-. Esto es causa de ansiedad: -Lo duro es el hecho de encadenarse a otra pastilla más, ese es el camino-, -... lo malo es que esto es para siempreTambién, hay pacientes que admiten no tomarla como les ha sido prescrita: -... a mí algún día se me olvidaLos pacientes de este estudio también manifiestan temores a la posible interacción con otros tratamientos. Esto indica que tanto el conocimiento erróneo, como algunas creencias y actitudes sobre los medicamentos pueden reducir el cumplimiento. Por lo tanto, el personal sanitario debe proporcionar información adecuada acerca de los efectos secundarios, interacciones medicamentosas en pluri-patologías y beneficios derivados de los fármacos antihipertensivos. De este modo se reduciría el miedo y la ansiedad derivada de su uso.

Por otro lado, Bane et al. (27) exponen que el $84.9 \%$ de los pacientes reportaron fuertes creencias sobre posibles efectos adversos con el uso de los medicamentos; es decir, que la adherencia se ve afectada por las creencias sobre el peligro de la dependencia y la toxicidad a largo plazo de los medicamentos. Otros estudios hacen referencia a los horarios de los medicamentos y la claridad del personal de salud para explicar dudas; que sólo el $8 \%$ de los pacientes presenta riesgo moderado para no adherirse, este porcentaje está relacionado con la orientación sobre la forma de ajustar los horarios de los medicamentos ordenados. Lo que significa que debe existir disponibilidad del equipo de salud organizado y que responda con claridad a las inquietudes relacionadas con la enfermedad y los tratamientos farmacológicos y no farmacológicos.

De modo similar, Allen et al. (28) reportan un cambio negativo en las creencias con respecto a los tratamientos a largo plazo en las enfermedades cardiovasculares. Estas creencias afectan la adherencia a los tratamientos debido a que los pacientes identifican signos y síntomas adicionales o efectos secundarios con los tratamientos y la dejan. En otro estudio se encontró que los participantes no les gustaba el tratamiento y sus efectos secundarios y se teme la adicción. En otros estudios los participantes confirmaron que su deseo de estar sanos era el principal motivador en la decisión de tomar su medicación; en este sentido, los participantes manifestaron querer sentirse bien, estar vivos, estar fuera del hospital y tener una buena calidad de vida. Este hallazgo demuestra que una actitud positiva hacia la vida aumenta la adherencia y que muchos pacientes con Enfermedades Cardiovasculares están motivados a cumplir para evitar hospitalizaciones (29). 


\section{Prácticas frente al tratamiento en ECV}

Los pacientes realizan prácticas como coadyuvantes para el manejo de su enfermedad, tales como la autoayuda, siempre influenciados por la cultura, como por ejemplo el uso de plantas medicinales, entre las cuales están: la zarzaparrilla, la cual tiene propiedades diuréticas y compuestos espasmolíticos que relajan las paredes arteriales, con una menor respuesta a la norepinefrina, la serotonina y las prostaglandinas; la natre y la traica. Además de nombrar las plantas medicinales en este estudio los pacientes dan a conocer cómo las preparan y su dosificación a través de infusiones y varias veces al día.

Nigenda et al. (30) encontraron que dentro de los tipos de medicina alternativa que utilizan los adultos mayores con enfermedades cardiovasculares están: la homeopatía, la acupuntura, la herbolaria, la masoterapia, el reiki, la magnetoterapia, la aromaterapia, la sonoterapia, la curación del daño, los suplementos dietéticos y la cirugía psíquica. Los autores definen las prácticas alternas "como un conjunto de conocimientos y experiencias generadas en el seno de la comunidad, transmitidos generacionalmente, basados en un saber fundamentalmente empírico, que intenta ofrecer soluciones a las diversas manifestaciones de la enfermedad, buscando propiciar la salud". Así mismo, manifiestan que en México las prácticas alternativas tienen gran arraigo cultural, pero que son muy costosas, además se trasmiten generacionalmente; sin embargo, no se conoce la prevalencia del uso ni sus efectos adversos.

Arteaga (31) en su estudio en la Pampa Argentina demuestra que actualmente los tratamientos que involucran prácticas y saberes tradicionales son el curanderismo y el autotratamiento o medicina casera; estas prácticas tienen un origen y arraigo popular, son terapéuticas rituales y concepciones de la salud de origen católico o refiguradas en términos de tales creencias religiosas. La medicina casera o autotratamiento es muy utilizado en Argentina, dentro de la praxis de la Medicina Casera; en este sentido, el autor habla de la Auto-atención, que incluye el consumo de fármacos de laboratorio y recetas tradicionales de la medicina casera que va desde infusiones, hasta aplicación de parches, cataplasmas, pasando por baños de asiento.

Estas prácticas las puede ejecutar el enfermo individualmente o con la ayuda de familiares. En algunas culturas existen los legos o practicantes y conocedores de terapéuticas particulares, son las personas que ejercen el oficio de tratantes, quienes tienen un conocimiento amplio de esta medicina y son referenciados por los miembros de la comunidad. Estos legos usan plantas medicinales y toda clase de productos vegetales; igualmente, tratan todo tipo de enfermedades desde fracturas hasta anginas, no cobran dinero por su trabajo sólo hay una reciprocidad que el cliente por agradecimiento ofrece al tratante. Estos saberes se transmiten de generación en generación. Con respecto a las preparaciones van desde pomadas, remedios -infusiones, cataplasmas, bálsamos. Es de hacer notar, que las técnicas terapéutico-rituales que utilizan manipulan el poder, en términos de sagrado, trayendo al presente las acciones de personajes míticos o seres sagrados del cristianismo. Algunos tratamientos alternativos que los pacientes usan para el manejo casero de su enfermedad son el té de alpiste, la oliva, el té y las pastillas de ajo.

\section{Relación profesional-paciente}

Está bastante documentado que la relación profesionalpaciente es importante en la adherencia de estos a $\mathrm{su}$ tratamiento farmacológico y no farmacológico. Conviene subrayar sobre la importancia que tiene el tiempo que el funcionario dedica para hablarle al paciente de los horarios, de los posibles efectos secundarios, de las interacciones, de la consejería, de estilos de vida saludables, del ejercicio y de la calidad; como mínimo debe explicarle tres veces a la semana, sin dejar de lado de prevenirlo contra el consumo del alcohol y el tabaco. Sin embargo, en los estudios revisados los pacientes manifiestan que la información es demasiado general y no se adaptada a sus necesidades.. Así mismo, señalan que no poseían información sobre los beneficios que, sobre la salud en general y sobre su patología en particular, se derivaban al realizar una actividad física de forma continuada. Este estudio concluye que sería necesario que el personal sanitario explicase claramente el porqué de las medidas higiénico-dietéticas (dieta y actividad física) y las adaptase a cada paciente en particular.

Todo parece confirmar, que la clave para que los pacientes se adhieran a los tratamientos es mantener una relación positiva entre los profesionales de la salud y sus pacientes. Quienes tienen una relación positiva con los profesionales del cuidado de la salud, manifiestan seguir con él porque les genera confianza, 
ISSN-PRINT

1794-9831

E-ISSN 2322-7028

Vol. 15 No. 2

Jul - Dic 2018

Cúcuta, Colombia respeto y experiencia. Por el contrario, los que tienen una mala relación con los proveedores de cuidado de la salud no se sienten obligados a adherirse (32).

Los pacientes se adhieren más fácilmente a los tratamientos farmacológicos cuando los síntomas de los efectos secundarios son mínimos. También se encontró que las barreras más sobresalientes al tomar la medicación están relacionadas con los horarios, el número de píldoras y la dificultad para tragar los medicamentos. Con estos hallazgos se concluye que la polifarmacia contribuye a la no adherencia y que el conocimiento sobre los medicamentos debe estar basado en las necesidades individuales de cada persona. La mayoría de los participantes identificaron olvidar tomar el medicamento como una barrera crucial para el cumplimiento de la medicación. El olvido ha sido bien documentado en otros estudios (33). La capacidad del paciente para abrir y extraer las tabletas de paquetes comerciales comunes o contenedores, son otras barreras contra la adherencia a los tratamientos; igualmente están relacionadas con problemas cognitivos y de fuerza en pacientes con enfermedad cardiovascular.

Otro aspecto importante a tener en cuenta en la comunicación paciente - profesional de la salud es que estos sobre-estiman la capacidad de los pacientes para entender las explicaciones, sobre todo en pacientes de mayor edad. En definitiva, se concluye que la relación profesional paciente debe ser nutrida, para que el paciente se adhiera a los tratamientos; también debe ser individualizada y adaptada a las necesidades de cada paciente.

\section{Papel del contexto en la trasmisión de creencias y prácticas en ECV}

La familia constituye la palanca fundamental en la transmisión de las creencias, de la religión y de los saberes en general. González y Fontao (34), hallaron que la familia se constituye en el principal medio de socialización de las creencias y normas de conductas propias de la cultura popular hacia todos sus miembros, sobre todo en los más jóvenes; es por eso, que el ambiente familiar y el contexto social en que la persona crece es importante para adquirir hábitos o desecharlos. Es decir, que la adherencia terapéutica se asocia a la presencia de suficientes redes de apoyo sociales. En la adherencia terapéutica tiene un alto significado el apoyo que el paciente recibe de familiares y amigos; contribuyendo a incrementar la ejecución de las prescripciones terapéuticas; igualmente puede animar al enfermo a mantener el régimen médico. Louro Bernal citado por La Rosa et al. (35) plantea que la familia constituye la primera red de apoyo social que posee el individuo, ejerciendo una función protectora ante las tensiones que genera la vida cotidiana. El apoyo que ofrece la familia es el principal recurso de promoción de la salud, así como de la prevención de la enfermedad y sus daños. De igual forma, se constituye en el apoyo más eficaz que siente y percibe el individuo frente a todos los cambios y contingencias a lo largo del ciclo vital en el contexto social.

Del mismo modo, Espitia y Rincón (36), en un estudio con pacientes de evento coronario agudo, confirmaron que la influencia de la familia es muy importante porque allí se aprende por medio del ejemplo y de la práctica en el diario vivir. Es la familia quien incentiva a los pacientes para que se tomen los medicamentos o un alimento sano; pero es también la familia la que transmite de generación en generación los malos hábitos que se adquieren en los eventos sociales como fiestas y celebraciones donde está comprometido el consumo de alcohol, de comidas ricas en grasa y de tabaco; prácticas que inducen a factores de riesgo. El apoyo de la familia debe actuar como una señal de vida para recordar a los pacientes a tomar sus medicamentos en los horarios indicados; por lo tanto, es muy importante ayudar a los pacientes a desarrollar el hábito de tomar su medicación siguiendo las instrucciones de los especialistas.

Por su parte, Leonga et al. (37) recomiendan que las familias también deben formar parte de programas de rehabilitación cardiaca, ya que pueden influir en la adhesión y en las creencias o características de personalidad del paciente. La adherencia debe ser evaluada en pacientes con infarto de miocardio con el fin de identificar quién tiene más probabilidades de ser adherente y así proporcionar un apoyo más individualizado. Debe señalarse, que estas prácticas son transmitidas de generación en generación y que es la mujer la que se encarga de transmitirlas en un mayor porcentaje.

\section{Relación de las creencias y prácticas con la adherencia al tratamiento}

Los pacientes conECV se deben adherir a los tratamientos no farmacológicos como la consejería sobre estilos 
de vida saludables, la dieta, el ejercicio, el consumo de alcohol y tabaco. Sin embargo, esto se ve afectado por las creencias que los pacientes tienen respecto a este aspecto. Se ha demostrado que algunos pacientes nunca se adhieren al tratamiento no farmacológico, porque nunca siguen las instrucciones acerca de sus hábitos alimenticios, ni hacen ejercicio al pensar que les aumenta la presión arterial. Otros persisten en realizar conductas no saludables después del diagnóstico de su enfermedad como incremento de sodio en las comidas, consumo regular de ácidos grasos y alcohol, así como la falta de ejercicio físico regular (38-42).

Por otro lado, Gómez et al. (43) en su estudio sobre factores de riesgo y autovaloración del paciente en un primer episodio de cardiopatía isquémica, demostraron que los pacientes tienen ciertas creencias frente a los hábitos de vida: el $36 \%$ eran fumadores; el $24 \%$ consideró que el tabaco no constituía un riesgo para su salud. Un $32 \%$ presentaba un consumo excesivo de alcohol. El $75 \%$ consideró que el alcohol era una sustancia vitalizante. Un $35 \%$ eran obesos y un 72,4 $\%$ tomaba una dieta grasa; sólo el $7 \%$ consideró que su dieta no era adecuada o muy adecuada. El $60 \%$ eran sedentarios, el $62 \%$ manifestó haber sufrido estrés en los últimos 6 meses y un $85 \%$ se consideró satisfecho con su estilo de vida. Sin embargo, el 73,7 \% creía que la cardiopatía isquémica alteraría mucho sus actividades de la vida cotidiana y que los cambios más difíciles serían la alimentación, el tabaco y el ejercicio físico.

Con relación a los estilos de vida saludables como la dieta, el consumo de alcohol y el tabaco, Acosta et al. (44) demostraron que de los 165 pacientes que encuestaron no asocian su enfermedad cardiovascular con los siguientes factores de riesgo: el consumo de sal $63.7 \%$, consumo de alcohol $96.3 \%$, el hábito de fumar $57.6 \%$, el consumo de grasa saturada $48.4 \%$, el sedentarismo $88,4 \%$ y la obesidad $66.0 \%$. Se debe destacar, que el acto de fumar es una actividad social; es decir, que algunos pacientes identificaron "el consumo como destino, es decir, que aun conociendo los daños que el consumo de cigarrillo puede causar en la salud, se continua con la práctica de fumar y está sumada a lo que ha sido llamado como inminencia de muerte, en atención a que hay plena conciencia del daño causado, pero se cambia ese conocimiento, por la sensación de placer, o de saber que para morir es necesario estar vivos". También consideraron el alcohol como coadyuvante del tabaco en un $40 \%$, y acompañado de cafeína un $60 \%$.
Por otro lado, llama la atención que este grupo no tiene establecido, en sus creencias, la percepción individual sobre los riesgos de la enfermedad tan necesarios en el logro de la adherencia al tratamiento, ya que a mayor percepción de vulnerabilidad personal se asocia un mayor interés en el aprendizaje y ejecución de conductas preventivas (45-46).

En el estudio de Rodríguez y Car (47) se evidenciaron percepciones que acentúan el riesgo sobre todo con cada cambio de presión. Diversos sentimientos emergen como una manifestación de la nueva realidad que incluyen el miedo a la muerte, complicaciones de salud, así como el temor a nuevas hospitalizaciones. Por su parte, Espitia y otros investigadores encontraron en pacientes que presentaron un Infarto Agudo de Miocardio reportes sobre una vivencia traumática que no se quiere volver a repetir por su connotación de insoportable; de ahí que sea necesario resaltar, que esta huella o señal que deja la experiencia de sufrir una enfermedad coronaria, con su inevitable riesgo de dolor y muerte, es determinante en la adopción del cambio de hábitos. De igual forma, Debs et al. (48) en su estudio encontraron que más de la mitad de los encuestados no conocía ningún factor que precipitara el desarrollo de una presión arterial alta 52,3 $\%$, ni cómo prevenirla $65,4 \%$.

\section{Rol del enfermero}

El papel del profesional de enfermería es fundamental para que los pacientes con enfermedades cardiacas se adhieran a los tratamientos. Definitivamente la herramienta más importante de estos profesionales es la educación, pero ante todo conocer al paciente para poder ofrecerle educación individualizada de acuerdo a sus necesidades y creencias.

Cornélio et al. (49) enfatizan "En el desarrollo de escalas para mensurar los factores que subsidian el comportamiento de consumo de sal, principalmente en hipertensos, que se benefician significativamente con la adopción de una dieta con bajo contenido de sal, estos ejercicios investigativos tienen implicaciones para la práctica educativa del enfermero". Medidas confiables y válidas son necesarias para que el enfermero pueda crear y evaluar programas de orientación basados en teorías provenientes de las ciencias sociales y del comportamiento; de tal forma que tengan mayor probabilidad de efectividad.

Es importante resaltar el papel que deben desempeñar todos los profesionales de la salud, teniendo en cuenta, $\overline{\text { E-ISSN 2322-7028 }}$

Vol. 15 No. 2

Jul - Dic 2018

Cúcuta, Colombia 
ISSN-PRINT

1794-9831

E-ISSN 2322-7028

Vol. 15 No. 2

Jul - Dic 2018

Cúcuta, Colombia que el tratamiento de estos pacientes es interdisciplinar; razón por la cual tanto el médico como el enfermero deben tener las competencias necesarias para ofrecer intervenciones de calidad.

Por otro lado, Flores (50) señala que el médico de familia, por su proximidad al paciente hipertenso, así como al conocimiento de su entorno familiar y social, se encuentra en un lugar privilegiado, puesto que le permite desarrollar intervenciones desde un enfoque biopsicosocial, atendiendo a los hábitos cotidianos, las necesidades, las preferencias y los antecedentes particulares de cada individuo. Desde esta perspectiva, debe elegir estrategias que atraigan la participación activa del paciente en el proceso de autocuidado, de tal forma que favorezcan su adherencia a los regímenes prescritos y permitan un esfuerzo continuo y periódico en los logros alcanzados. Así mismo, especifica que la investigación representa un aporte especial a la práctica de enfermería, porque permite elevar cualitativamente el rol profesional y fortalecer el prestigio de la enfermera a partir de una mejora en su desempeño, permitiéndole retomar una de sus funciones específicas, como es la educación.

También, es importante que el enfermero (a) identifique las creencias y las prácticas que los pacientes tienen para poder individualizar las intervenciones. Igualmente, debe identificar las creencias no generadoras de salud de los pacientes para intentar corregirlas y una vez reconocidas utilizarlas para lograr cambios en las conductas que lleve al logro de una verdadera medicina preventiva. De ahí, que sea necesario conocer las medidas que corrijan la presencia de estos factores proponiendo estrategias acordes al contexto en el que el paciente se desenvuelve.

Para concluir, es importante resaltar que esta categoría de análisis enriquece el tema ya que, en la medida en que se pueda negociar con la persona sobre sus gustos y direccionarlos en pro de la vida sana, sin atropellar su deseo, se pueden obtener mejores resultados. Igualmente, se debe enfatizar en que el conocimiento por sí solo no es suficiente para cambiar el comportamiento. Es muy importante, comprender las creencias de una persona y las actitudes acerca de su riesgo de enfermedad cardiovascular, a fin de que el profesional pueda adaptar la información a los pacientes, de tal forma que pueda persuadir a los individuos a adoptar estilos de vida saludables, mediante respuestas adecuadas.

\section{Conclusiones}

- Según la Organización Mundial de la Salud los porcentajes de adherencia son bajos y están en un promedio de $50 \%$; dicha adherencia se ve afectada por muchos factores entre los que se encuentran las creencias que los pacientes tienen

- Frente a la enfermedad y a sus tratamientos farmacológicos y no farmacológicos -como la nutrición, el consumo de alcohol y tabaco, así como la baja adherencia al ejercicio-, las prácticas que realizan como coadyuvantes para los tratamientos tradicionales tales como el uso de plantas medicinales, infusiones, rituales, entre otros, están muy arraigados en la población porque hacen parte de los saberes que se transmiten de generación en generación, saber que no se puede desconocer.

- Muchas veces por ignorancia y por falta de respeto hacia las creencias y prácticas de los pacientes con enfermedad cardiovascular, los profesionales no tienen en cuenta la individualidad y el contexto en el que viven ya sea para ajustar el tratamiento o para brindar una consejería apropiada que resuelva las dudas y haga al paciente partícipe y no solo espectador de su propia vida; de ahí, que las recomendaciones sobre estilo de vida, medicación, efectos secundarios, son lejanas a las personas, distantes a su entorno y vida real y sobre todo a las familias.

- Es necesario ver al paciente en su individualidad y como integrante de una sociedad y una cultura, que han influido en sus creencias y prácticas de cuidado, por tanto, desde la enfermería se debe aplicar un cuidado transcultural. Reconocer que las prácticas y saberes no tradicionales como el curanderismo y la medicina casera, son prácticas que tienen un origen y arraigo popular, es una forma de acercarse a las comunidades y sus conocimientos. Entender estas creencias y prácticas ayudará al enfermero (a) a diseñar intervenciones más efectivas y cercanas a las realidades de los pacientes.

\section{Conflicto de Intereses}

La autora declara no tener ningún conflicto de intereses. 


\section{Referencias Bibliográficas}

1. Organización Mundial de la Salud. Enfermedades Cardiovasculares. [Internet] 2015 [Consultado el 20 de agosto de 2017]; Disponible: http://www.who.int/mediacentre/factsheets/fs317/es/

2. DANE, Estadísticas vitales, diez primeras causas de defunción, según departamento de residencia, [Internet]. [Consultado el 20 de agosto de 2017]; Disponible en: http://www.dane.gov.co/files/ investigaciones/poblacion/defunciones/defun 2008/cuadro5.xls.

3. Organización Mundial de la Salud. Adherencia a los tratamientos a largo plazo. Pruebas para la acción. Capitulo V. [Internet]. 2008 [Consultado el 20 de agosto de 2017]. Disponible en: http://www.amro. who.int/common/Display.asp?Lang=S\&RecID=8062.

4. Martín Alfonso L. Repercusiones para la salud pública de la adherencia terapéutica deficiente. Salud Pública. Rev Cubana Salud Pública. [Internet]. 2006 [Consultado el 20 de agosto de 2017]; 32(3). Disponible en: http://scielo.sld.cu/scielo.php?script=sci arttext\&pid=S0864-34662006000300013

5. Organización Mundial de la Salud. Alerta del bajo seguimiento de los tratamientos por parte de pacientes con enfermedades crónicas. Madrid: Ediciones Doyma. [Internet]. 2003 [Consultado el 20 de agosto de 2017]. Disponible en: http://www.who.int/mediacentre/news/releases/2003/pr54/es/

6. Organización Mundial de la Salud. Adherencia a los tratamientos a largo plazo. Pruebas para la acción. Capítulo I. [Internet]. 2004 [Consultado el 20 de agosto de 2017]. Disponible en: http://www.amro. who.int/common/Display.asp?Lang=S\&RecID=8062.

7. Agámez Paternina AP, Hernández Riera R, Cervera Estrada L, Rodríguez Gracia Y. Factores relacionados con la no adherencia al tratamiento antihipertensivo. ACM [Internet]. 2008 [Consultado el 20 de agosto de 2017]; 12(5). Disponible en: http://scielo.sld.cu/pdf/amc/v12n5/amc09508.pdf

8. Sánchez Cisneros N. Adherencia terapéutica en hipertensión arterial sistémica. Revista Mexicana de Enfermería Cardiaca. [Internet]. 2006 [Consultado el 23 de agosto de 2017]; 14(3):88-101. Disponible en: http://www.medigraphic.com/pdfs/enfe/en-2006/en063d.pdf

9. Real Academia Española. Diccionario de la lengua española. Vigésima segunda edición. [Internet]. [Citado el 23 de agosto de 2017]. Disponible en: http://dle.rae.es/srv/fetch?id=TtEMsxJ

10. Márquez Contreras E, Casado Martínez JJ, de la Figuera Won Vichman M, Gil Guillén V, Martell N. El incumplimiento terapéutico en el tratamiento de la hipertensión arterial en España. Análisis de los estudios publicados entre 1984 y 2001. Hipertensión y Riesgo Vascular [Internet]. 2002 [Consultado el 23 de agosto de 2017]; 19(1):12-16. Disponible en: http://www.elsevier.es/es-revista-hipertensionriesgo-vascular-67-articulo-el-incumplimiento-terapeutico-el-tratamiento-S1889183702712166

11. Granados G, Roales JG, Moreno E, Ybarra JL. Creencias en síntomas y adherencia al tratamiento farmacológico en pacientes con hipertensión. (Spanish). International Journal Of Clinical Health \& Psychology. [Internet]. 2007 [Consultado el 23 de agosto de 2017]; 7(3):697-707. Disponible en: http://promsalud.udenar.edu.co/wpcontent/uploads/2014/08/adhesion-al-tratamiento-y-creencias-en-sintomas.pdf

12. Giñarte Arias Y. La adherencia terapéutica. Rev Cubana Med Gen Integr [Internet]. 2001 [Consultado el 23 de agosto de 2017]; 17(5):502-505. Disponible en: http://scielo.sld.cu/scielo.php?script=sci_artt ext\&pid $=$ S0864-21252001000500016

13. Orueta Sánchez R. Estrategias para mejorar la adherencia terapéutica en patologías crónicas. Información Terapéutica del Sistema Nacional de Salud. [Internet]. 2005 [Consultado el 25 de agosto de 2017]; 2(29):40-45. Disponible en: https://www.msssi.gob.es/biblioPublic/publicaciones/docs/ vol29 2EstrategiasMejora.pdf

14. Schmidt Rio Valle J, Cruz Quintana F, Villaverde Gutiérrez C, Prados PeñaD, García Caro MP, etal. Treatment adherence of hypertensive patients: a qualitative study. Index Enferm. [Internet]. 2006 [Consultado el 25 de agosto de 2017]; 15(54):25-29. Disponible en: http://dx.doi.org/10.4321/S1132-12962006000200005

15. Herrera Lián A. Factores que influyen en la adherencia a tratamientos en pacientes con riesgo de 
ISSN-PRINT

1794-9831

E-ISSN 2322-7028

Vol. 15 No. 2

Jul - Dic 2018

Cúcuta, Colombia

enfermedad cardiovascular. Avances en Enfermería. [Internet]. 2008 [Consultado el 25 de agosto de 2017]; 26(1):36-42. Disponible en: http://revistas.unal.edu.co/index.php/avenferm/article/view/12883

16. Arrieta Pérez RT, Garfías Jiménez F. Beliefs about the Origin of the Risk Factors for Suffering from Hypertension in Hypertensive Mexican Group Patients. Archivos en Medicina Familiar. [Internet]. 2010 [Consultado el 25 de agosto de 2017]; 12(2):57-64. Disponible en: http://www.medigraphic. com/pdfs/medfam/amf-2010/amf102e.pdf

17. Jia Rong W, Moser DK, Lennie TA, Peden RC, Yu Chang, Seongkum H. Factors influencing medication adherence in patients with heart failure. Heart Lung [Internet]. 2008 [Consultado el 25 de agosto de 2017]; 37(1):8-16. Disponible en: https://www.ncbi.nlm.nih.gov/pubmed/18206522

18. Marshall IJ, Wolfe CD, McKevitt C. Lay perspectives on hypertension and drug adherence: systematic review of qualitative research. BMJ. [Internet]. 2012 [Consultado el 25 de agosto de 2017]; 345:1-16 Disponible en: https://www.ncbi.nlm.nih.gov/pubmed/22777025

19. González González BA, Zarza Arizmendi MD, Zárate Grajales RA, Salcedo Álvarez RA, Alba Leone A, Rubio Domínguez S. Adultos mayores, calidad de vida y uso de medicina científica con prácticas alternas. México 2007. Revista CONAMED. [Internet]. 2008 [Consultado el 25 de agosto de 2017]; 13(2):23-29. Disponible en: https://dialnet.unirioja.es/descarga/articulo/3625212.pdf

20. Muñoz LA, Price Y. Comprendiendo la búsqueda del bienestar en pacientes del programa de hipertensión Arterial. Investigación y Educación en Enfermería [Internet]. 2003 [Consultado el 25 de agosto de 2017] 21(1):38-46. Disponible en: https://dialnet.unirioja.es/servlet/articulo?codigo=1464617

21. Faleiros Taveira L, Geraldo Pierin AM. ¿Puede el nivel socioeconómico influenciar las características de un grupo de hipertensos? Rev Latino-am Enfermagem. [Internet]. 2007 [Consultado el 25 de agosto de 2017]; 15(5). Disponible en: http://www.scielo.br/pdf/rlae/v15n5/es_v15n5a07.pdf

22. Dos Santos JE, De Oliveira Augusto MA, Gusmao J, Décio Mion J, Décio, Ortega K, Geraldo Pierin AM. Profile of hypertensive patients: biosocial characteristics, knowledge, and treatment compliance. Acta paul. enferm. [Internet]. 2008 [Consultado el 25 de agosto de 2017]; 21(1):59-65. Disponible en: http://dx.doi.org/10.1590/S0103-21002008000100009

23. Roales Nieto JG, Ybarra Sagarduy JL, Granados Gámez G. Un estudio exploratorio sobre el desarrollo de creencias sobre síntomas como señales de hipertensión arterial. Psicothema. [Internet]. 2006 [Consultado el 25 de agosto de 2017]; 18(4):822-827. Disponible en: http://www.psicothema.com/psicothema.asp?id=3315

24. Silberman P, Ruggero S, Perruza F, López S, Polla S, et al. Saberes previos y adherencia terapeútica en hipertensión. Rev. Asoc. Med. Bahía Blanca [Internet]. 2006 [Consultado el 25 de agosto de 2017]; 16(2):46-50. Disponible en: http://pesquisa.bvsalud.org/portal/resource/pt/bin-120881

25. Mendoza Montano C, Estrada K, Chávez A, Ramírez Zea M. Perceptions, knowledge and beliefs about prevention of cardiovascular diseases in Villa Nueva, Guatemala. Prevention and Control. [Internet]. 2008 [Consultado el 25 de agosto de 2017]; 3(1):1-9. Disponible en: https://www.researchgate.net/ publication/244821411_Perceptions knowledge and beliefs about prevention_of cardiovascular diseases in Villa_Nueva_Guatemala

26. Frick U, Gutzwiller FS, Wiedermann W. A questionnaire on treatment satisfaction and disease specific knowledge among patients with acute coronary syndrome. II: Insights for patient education and quality improvement. Patient Education and Counseling. [Internet]. 2012 [Consultado el 25 de agosto de 2017]; 86(3):366-371. Disponible en: https://www.ncbi.nlm.nih.gov/pubmed/21719236

27. Bane C, Hughes CM, McElnay JC. The impact of depressive symptoms and psychosocial factors on medication adherence in cardiovascular disease. Patient Education and Counseling. [Internet]. 2006 [Consultado el 25 de agosto de 2017]; 60(2):187-193 Disponible en: http://www.sciencedirect.com/ science/article/pii/S0738399105000066

28. Allen LaPointe NM, Fang Shu Ou, Calvert S. B, Melloni C, Stafford JA, Harding T, Alexander KP, Peterson ED. Changes in beliefs about medications during long-term care for ischemic heart disease. 
American Heart Journal. [Internet]. 2010 [Consultado el 25 de agosto de 2017]; 159(4):561-569. Disponible en: https://www.ncbi.nlm.nih.gov/pubmed/20362713

29. Rodríguez Acelas AL, Gómez Ochoa AM. Factores influyentes en adherencia al tratamiento en pacientes con riesgo cardiovascular. Avances en Enfermería. [Internet]. 2010 [Consultado el 25 de agosto de 2017]; 28(1):63-71. Disponible en: http://www.revistas.unal.edu.co/index.php/avenferm/article/view/15656/18159

30. Nigenda G, Mora G, Ruíz JA, Céspedes J. Modelos alternativos de atención a la salud: utilización y disponibilidad en la Ciudad de México. [Internet]. 2002 [Consultado el 25 de agosto de 2017] 1-52. Disponible en: https://www.yumpu.com/es/document/view/14772431/modelos-alternativos-deatencion-a-la-salud-utilizacion-y-funsalud

31. Arteaga F. Rituales y remedios naturales: Las prácticas del autotratamiento en la Pampa (Argentina). Mitológicas [Internet]. 2010 [Consultado el 25 de agosto de 2017]; 25:9-26. Disponible en: http:// www.redalyc.org/pdf/146/14615247001.pdf

32. Stromberg A, Brostrom A, Dahlstrom U, Fridlund B. Factors influencing patient compliance with therapeutic regimens in chronic heart failure: a critical incident technique analysis. Heart Lung 1999; 28(5):334-41.

33. Evangelista L, Doering LV, Dracup K, Westlake C, Hamilton M, Fonarow GC. Compliance behaviors of elderly patients with advanced heart failure. J Cardiovasc Nurs. [Internet]. 2003 [Consultado el 25 de agosto de 2017]; 18(3):197-206. Disponible en: https://www.ncbi.nlm.nih.gov/pubmed/12837010

34. González Consuegra RV, Fontao Zago MM. Creencias en fumadores pertenecientes a un programa de salud cardiovasculas. Rev. Latino-Am. Enfermagem. [Internet]. 2004 [Consultado el 25 de agosto de 2017]; 12:412-419. Disponible en: http://dx.doi.org/10.1590/S0104-11692004000700017

35. La Rosa Matos Y, Martín Alfonso L, Bayarre Vea H. Adherencia terapéutica y factores psicosociales en pacientes hipertensos. Rev Cub de Med Gen Integr. [Internet]. 2007 [Consultado el 25 de agosto de 2017]; 23(1). Disponible en: http://scielo.sld.cu/scielo.php?script=sci_arttext\&pid $=\mathrm{S} 0864-21252007000100005$

36. Espitia Cruz SE, Rincón Osorio F. Percepciones y creencias en personas que padecieron un evento coronario agudo. Av.enferm. [Internet]. 2011 [Consultado el 25 de agosto de 2017]; 29(2):307-318. Disponible en: http://revistas.unal.edu.co/index.php/avenferm/article/view/35825/37092

37. Leonga J, Molassiotisa A, Marsh H. Adherence to health recommendations after a cardiac rehabilitation programme in post-myocardial infarction patients: the role of health beliefs, locus of control and psychological status. Clinical Effectiveness in Nursing. [Internet]. 2004 [Consultado el 25 de agosto de 2017]; 8(1):26-38. Disponible en: http://www.sciencedirect.com/science/article/pii/S1361900404000032

38. Furtado De Lima e Costa MF, Peixoto Sérgio V, Comini César C, Carvalho Malta D, De Moura EC. Health behaviors among older adults with hypertension, Brazil, 2006. Rev Saude Publica [Internet]. 2009 [Consultado el 25 de agosto de 2017]; 43(2):18-26. Disponible en: http://dx.doi.org/10.1590/ $\underline{\mathrm{S} 0034-89102009000900004}$

39. DeAzevedo Barros MB, Znchetta LM, DeMoura EC, Carvalho Malta D. Self-rated health and associated factors, Brazil, 2006. Rev Saude Publica. [Internet]. 2009 [Consultado el 25 de agosto de 2017] 43(2):2737. Disponible en: http://www.scielo.br/scielo.php?pid=S0034-89102009000900005\&script=sci arttext\&tlng=en

40. Leite Cavalcanti C, Rodrigues Gonçalves MC, Rios Asciutti LS, Leite Cavalcanti A. Prevalência de doenças crônicas e estado nutricional em um grupo de idosos brasileiros. Rev Salud Publica. [Internet]. 2009 [Consultado el 25 de agosto de 2017]; 11(6): 865-877. Disponible en: http://www.scielosp.org/ scielo.php?script $=$ sci arttext\&pid $=$ S0124-00642009000600003

41. Oliveira Reiners AA, Correa da Costa Ribeiro AL, DeArruda Gawlinski AL, Nogueira Suely M. Hipertensão arterial: perfil de saúde dos trabalhadores de enfermagem de um hospital universitário. Texto \& contexto enferm. [Internet]. 2004 [Consultado el 25 de agosto de 2017]; 13(1):41-49. 
ISSN-PRINT

$1794-9831$

E-ISSN 2322-7028

Vol. 15 No. 2

Jul - Dic 2018

Cúcuta, Colombia

Disponible en: https://www.researchgate.net/publication/26603331_Hipertensao_arterial_perfil_de saude dos trabalhadores de enfermagem de um hospital universitario

42. Flores O. Hipertensión arterial y las modificaciones de los estilos de vida en la práctica familiar. Med. fam. (Caracas) [Internet]. 2001 [Consultado el 25 de agosto de 2017]; 9(2):137-142. Disponible en: http://pesquisa.bvsalud.org/portal/resource/pt/lil-350896

43. Gómez MJ, Gil D, Ortigosa L, Isla P, Vivas E, et al. Factores de riesgo y autovaloración del paciente en un primer episodio de cardiopatía isquémica. Enfermería clínica. [Internet]. 2003 [Consultado el 26 de agosto de 2017]; 13(5):267-278. Disponible en: http://www.elsevier.es/es-revista-enfermeria-clinica35-articulo-factores-riesgo-autovaloracion-del-paciente-S1130862103738200

44. Acosta González M, Debs Pérez G, De la Noval García R, Dueñas Herrera A. Conocimientos, creenciasy prácticas en pacienteshipertensos, relacionadosconsuadherenciaterapéutica. Revista Cubana de Enfermería. [Internet]. 2005 [Consultado el 26 de agosto de 2017]; 21(3):63-70 Disponible en: http://scielo.sld.cu/scielo.php?script=sci arttext\&pid $=\mathrm{S} 0864-03192005000300008$

45. Jensen LA, Moser DK. Gender Differences in Knowledge, Attitudes, and Beliefs About Heart Disease. Nursing Clinics of North America 2008; 43(1):77-104.

46. Caloto MT, Troya J, García E, Giménez G, Marcos G, et al. Attitudes and beliefs of patients with high cardiovascular risk, about hypercholesterolemia and its treatment in espain. The practice study. Value in Health. 2007; 10(6):A434.

47. Rodrigues Carrijo LM, Car MR. Dialética do modo de vida de portadores de hipertensão arterial: o objetivo e subjetivo. Rev Esc Enferm USP. [Internet] 2006 [Consultado el 23 de diciembre de 2017]; 41(4):573-580. Disponible en: http://www.ee.usp.br/reeusp/upload/pdf/757.pdf

48. Debs G, Noval R, Duenas A, Rodriguez F. Criteria, attitudes and beliefs about arterial hypertension among the population of the "10 de Octubre" municipality. Rev. cuba. med. gen. Integr. [Internet] 1996. [Consultado el 26 de agosto de 2017]; 12(2):120-125 Disponible en: http://pesquisa.bvsalud. org/portal/resource/pt/cum-8303

49. Cornélio ME, Bueno Jayme MC, Godin G, Matheus Rodrigues RC, DelaRosa Mendes R, Nadruz Junior W. Decelopment and reliability of an instrument to measure psychosocial determinants of salt consumption among hypertensive patients. Rev Lat Am Enfermagem. [Internet]. 2009 [Consultado el 26 de agosto de 2017]; 17(5):701-707. Disponible en: http://www.scielo.br/scielo.php?script=sci artt ext\&pid=S0104-11692009000500017

50. Flores Villegas R. Programa educativo dirigido a pacientes con hipertensión arterial sistémica en la consulta externa del Hospital Obrero Numero 1 gestión 2001. [Tesis en Internet]. La Paz: Universidad Mayor de San Andrés; 2001. 